\title{
The role of tendon and subacromial bursa in rotator cuff tear pain: a clinical and histopathological study
}

\author{
Claudio Chillemi $^{1} \cdot$ Vincenzo Petrozza $^{2}$ - Vincenzo Franceschini ${ }^{3} \cdot$ Luca Garro $^{4}$. \\ Alberto Pacchiarotti ${ }^{5} \cdot$ Natale Porta $^{2} \cdot$ Mirko Cirenza $^{2} \cdot$ Francesco Salate Santone $^{6}$. \\ Alessandro Castagna ${ }^{7}$
}

Received: 17 August 2014 / Accepted: 13 May 2015

(C) European Society of Sports Traumatology, Knee Surgery, Arthroscopy (ESSKA) 2015

\begin{abstract}
Purpose To evaluate a possible association of shoulder pain with the clinical features and the histopathological changes occurring in the ruptured tendon and subacromial bursa of patients with rotator cuff tear.

Methods One hundred and eighty patients were clinically evaluated with the constant score and the visual analogue pain scale. Radiographs and MRI were performed. The chronology of the rupture, the muscle fatty degeneration according to Goutallier's scale and the tear size were evaluated. For each patient, a biopsy of the supraspinatus tendon and subacromial bursa was performed during arthroscopic rotator cuff tear repair and the specimens were histopathologically analysed.

Results Clinically, the shoulder was more painful in females, in the presence of a chronic cuff lesion and a low Goutallier's grade $(P<0.05)$. No association was found between pain and age of the patient and between pain and
\end{abstract}

Claudio Chillemi

c_chillemi@libero.it

1 Department of Orthopaedic Surgery, Istituto Chirurgico Ortopedico Traumatologico (ICOT), Latina, Italy

2 Histology Unit, Department of Medico-Surgical Sciences and Biotechnologies, ICOT Latina, Sapienza University of Rome, Latina, Italy

3 Department of Orthopaedics and Traumatology, ICOT, Sapienza University of Rome, Latina, Italy

4 Department of Orthopaedic Surgery, University of Rome Tor Vergata, Rome, Italy

5 Department of Histopathology, Istituto Chirurgico Ortopedico Traumatologico (ICOT), Latina, Italy

6 Value Lab S.p.a, Rome, Italy

7 Istituto Clinico Humanitas IRCSS, Rozzano, MI, Italy tear size. Histologically, hypertrophy and inflammation of the tendon and hypertrophy, inflammation, oedema and necrosis of the subacromial bursa were directly associated with pain $(P<0.05)$. Pain decreased significantly in the presence of fatty metaplasia and necrosis of the tendon $(P<0.05)$.

Conclusions This study defines the main clinical and histopathological features of painful rotator cuff tear. In particular, a greater association of pain was observed with the histopathological changes in the bursa compared with those in the rotator cuff. Considering that the bursa plays also an essential role during the healing process, this "new" role of the subacromial bursa as pain generator has important repercussions in both pharmacological and surgical treatments of rotator cuff tears.

Level of evidence IV.

Keywords Histopathology - Pain · Subacromial bursa . Tendon $\cdot$ Rotator cuff tear

\section{Introduction}

Shoulder pain is the third (after back and neck pain) most common musculoskeletal complaint among the general population with almost $20 \%$ of people suffering from it during their lifetime $[8,15]$. Rotator cuff tear represents one of the most relevant causes of shoulder pain, with a prevalence (of both symptomatic and asymptomatic tears) ranging from 5 to $39 \%$ although higher rates are estimated for age $>50$ years [35].

The source of pain in rotator cuff tear is thought to be multifactorial. Pathologic changes have been observed in different structures of the shoulder. Tendon cells have been implicated as a potential source of inflammatory and pain 
mediators: increased levels of inflammatory and angiogenic cytokines have been observed in the torn tendon [10, 22] and in animal models of supraspinatus overuse and tendinopathy $[18,24]$. Inflammatory cytokines together with an increased number of afferent nerve endings have also been found in the subacromial bursa of patients with rotator cuff tears, suggesting that also this structure may play a role in the genesis of pain [5, 13, 26, 29, 33]. Degeneration of the long head of the biceps (LHB) tendon is often associated with massive rotator cuff tears and may contribute to shoulder pain and dysfunction $[6,30,36]$ as well as proximal humeral migration that is commonly observed in advanced rotator cuff tears [16].

It is clear that the causative factors responsible for pain in rotator cuff tear are poorly understood. In particular, there are no studies up to date that investigated the role of the histopathological changes occurring in the ruptured tendon and subacromial bursa in the genesis of pain. This observational laboratory study represents an attempt to clarify this unknown trying to associate pain with the clinical features and the histopathological changes observed in the torn tendon and subacromial bursa of patients with rotator cuff tears.

\section{Materials and methods}

Between 2009 and 2012, 460 consecutive patients underwent arthroscopic rotator cuff tear repair. One hundred and eighty patients, 78 men and 102 women, mean age 64.6 years \pm 6.2 , were included in the study. The inclusion criteria were as follows: the presence of a full thickness tear on MRI rotator cuff evaluation, no radiographic signs of fracture or osteoarthritis of the shoulder. The exclusion criteria were as follows: partial thickness tear, previous surgery in the affected shoulder, previous intraarticular injection therapy, history of shoulder instability or trauma, and shoulder stiffness. All the patients were initially treated with a standardized physical therapy protocol and underwent surgery because of functional impairment associated with pain.

Before surgery, all the patients were clinically evaluated with the constant score and the visual analogue scale (VAS) for pain. The latter consists of an unnumbered horizontal line, $10 \mathrm{~cm}$ in length, anchored by two verbal descriptors: "no pain" (score of 0) and "worst imaginable pain" (score of 10). The score was determined by measuring in $\mathrm{cm}$ the distance on the $10 \mathrm{~cm}$ line between the "no pain" anchor and the patient's mark with an accuracy of one decimal place. The VAS score obtained was a mean of the values reported for night pain, pain at rest and pain during daily's activities. Taking into account that pain is a subjective experience and each person has a threshold different from everyone else, patients were divided into three groups considering the VAS evaluation: Group A: $0 \leq \mathrm{VAS} \leq 5$; Group B: $5.5 \leq \mathrm{VAS} \leq 7.5$; and Group C: $8 \leq$ VAS $\leq 10$. Each patient was studied with a standardized imaging protocol: radiographs in four projections (AP in three rotations and outlet view) and MRI evaluation.

The chronological appearance of the rupture (meant as time from the onset of symptoms) was evaluated dividing the study population into three groups: Group 1: 0-6 months; Group 2: 6 months-1 year; and Group 3: $>1$ year. The muscle fatty degeneration of the rotator cuff was evaluated with MRI and graded, according to the classification system of Goutallier [12] modified by Fuchs et al. [9] for MRI in five stages (G-0: no fatty degeneration; G-1: muscle > fat; G-2: muscle $=$ fat; G-3: muscle $<$ fat; and G-4: complete replacement of muscle tissue with fat tissue).

A preliminary arthroscopic look was performed on each patients, before starting with the suture of the rotator cuff tear, to evaluate the tear pattern according to Snyder's classification system [28] which consider four types of lesions: $\mathrm{C} 1$ (lesion size $\leq 1 \mathrm{~cm}$ ), $\mathrm{C} 2$ (lesion width between 2 and $3 \mathrm{~cm}$ ), C3 (lesion width $>3 \mathrm{~cm}$ ) and $\mathrm{C} 4$ (massive retracted lesion). Without the use of epinephrine and before using any electric instruments (i.e. radiofrequency) for each patient, a biopsy of the edge of the ruptured supraspinatus tendon and subacromial bursa that cover the ruptured tendon was performed with a specific tool in the average size of $5 \times 5 \mathrm{~mm}$. The tissue material was fixed in neutral buffered formalin and paraffin-embedded according to standard procedures. Sections of $2 \mu \mathrm{m}$ were obtained and stained with haematoxylin-eosin (H/E), the gold standard staining for evaluating the morphology. The biopsy specimens of tendon and bursa were all subjected to histopathological analysis under light microscope and viewed by three different blinded pathologists.

All the histopathological features observed were scored into three groups according to a semiquantitative grading system: absent if the feature was not present in the specimen evaluated, focal if it was present only in few fields (max 3 fields at 10x) and small areas $(<10 \%$ of single field) and extended if it was present in more than three fields and in more than $10 \%$ of single field. In order to obtain an univocal estimate for each observed histopathological feature, the majority criterion was applied. All patients agreed to participate in the study and signed an informed consent for the use of data in accordance with the Declaration of Helsinki. All the procedures described in this study were approved by the Medical Ethical Committee of the "Humanitas Institute IRCSS Rozzano (MI), Italy". 


\section{Statistical analysis}

A statistical analysis was performed to examine the relationship between pain and clinical features of the patients and between pain and features of tendon and bursa. A Chi-square test was used to analyse the presence of significant association, while Cramer's V and gamma indexes estimated the strength of relationships. Cramer's V index attempts to quantify the strength of association between variables with no intrinsic order, and it varies between 0 and 1: close to 0 it shows no association between variables and close to 1 it indicates strong association. Instead, gamma index is a symmetric measure of association between two ordinal variables, and it ranges from -1 to 1 . Between quantitative and qualitative variables, a one-way analysis of variance (ANOVA) was performed. $P<0.05$ was considered statistically significant.

\section{Results}

The demographical data are reported in Table 1, while histopathological results of tendon and subacromial bursa are reported in Table 2. The mean preoperative constant score was $47.4 \pm 7.2$, and the mean score 18 months after surgery was $77.2 \pm 6.4(P=0.001)$.

Pain was significantly associated with female gender $(P=0.000, V=0.553)$, while there was no association with the age of the patients $(P=$ n.s.). In addition, pain was significantly associated with lower Goutallier's grades $(P=0.000)$. Pain was also directly associated with the time from the onset of symptoms being chronic lesions more painful than acute lesions $(P=0.007)$. Conversely, statistical analysis did not show any direct or inverse association between pain and size of the lesion $(P=$ n.s. $)$.

Taking into account the histopathological aspects observed (Fig. 1), a statistically significant association with pain was encountered for the following tendon features (Fig. 2): inflammation, hypertrophy, fatty metaplasia and necrosis $(P<0.05)$. The presence of inflammatory cells $(P=0.001)$ and hypertrophy/hyperplasia $(P=0.002)$ were significantly associated with pain, although the strength of these relationships was not particularly prominent (Cramer's $\mathrm{V}$ index was, respectively, equal to 0.414 and 0.387$)$. Instead, pain decreased significantly with fatty metaplasia $(P=0.000, V=0.437)$ and, to a lesser extent, with necrosis of the tendon $(P=0.031$, $V=0.288$ ).

A statistically significant association with pain was also detected for the following features of the subacromial bursa (Fig. 3): hypertrophy, inflammation, oedema and necrosis $(P<0.05)$. Pain was perceived in the presence
Table 1 Patients distribution according to the evaluated parameters

\begin{tabular}{|c|c|c|}
\hline & No of patients & $\%$ of patients \\
\hline \multicolumn{3}{|l|}{ Pain-VAS } \\
\hline A & 54 & 30 \\
\hline B & 63 & 35 \\
\hline $\mathrm{C}$ & 63 & 35 \\
\hline \multicolumn{3}{|c|}{ Onset of symptoms } \\
\hline Group 1 & 27 & 15 \\
\hline Group 2 & 96 & 53 \\
\hline Group 3 & 57 & 32 \\
\hline \multicolumn{3}{|c|}{ Fatty degeneration (Goutallier's scale) } \\
\hline GO & 30 & 17 \\
\hline G1 & 42 & 23 \\
\hline $\mathrm{G} 2$ & 45 & 25 \\
\hline G3 & 39 & 22 \\
\hline G4 & 24 & 13 \\
\hline \multicolumn{3}{|c|}{ Tear pattern (Snyder's classification) } \\
\hline $\mathrm{C} 1$ & 36 & 20 \\
\hline $\mathrm{C} 2$ & 90 & 50 \\
\hline $\mathrm{C} 3$ & 45 & 25 \\
\hline $\mathrm{C} 4$ & 9 & 5 \\
\hline \multicolumn{3}{|c|}{ Tendon involvement } \\
\hline SSP & 45 & 25 \\
\hline SSP, SSC & 27 & 15 \\
\hline SSP, ISP & 63 & 35 \\
\hline SSP, SSC, ISP & 45 & 25 \\
\hline
\end{tabular}

Pain was measured with the visual analogue scale (VAS), 0 being the inferior limit where patients were free of pain and 10 the superior limit of more painful shoulders (Group A: $0 \leq$ VAS $\leq 5$; Group B: $5.5 \leq$ VAS $\leq 7.5$; and Group C: $8 \leq$ VAS $\leq 10$ )

Onset of symptoms: The chronological appearance of the rupture was evaluated dividing the study population into three groups (Group 1: 0-6 months; Group 2: 6 months-1 year; and Group 3: >1 year)

Muscle fatty degeneration was graded, according to the classification system of Goutallier, in five stages (G-0: no fatty degeneration; G-1: muscle > fat; G-2: muscle = fat; G-3: muscle < fat; and G-4: complete replacement of muscle tissue with fat tissue)

Rotator cuff tear pattern was evaluated according to Snyder's classification system which considers four types of lesions: C1 (lesion size $\leq 1 \mathrm{~cm}$ ), C2 (lesion width between 2 and $3 \mathrm{~cm}$ ), C3 (lesion width $>3 \mathrm{~cm}$ ) and $\mathrm{C} 4$ (massive retracted lesion)

Tendon involvement: $\mathrm{SSP}=$ supraspinatus; ISP $=$ infraspinatus; $\mathrm{SSC}=$ subscapularis

of hypertrophy/hyperplasia $(P=0.000)$ and inflammatory cells $(P=0.000)$, as shown by the significant values of Cramer's V (respectively, equal to 0.796 and 0.580). Pain also increased significantly with bursal oedema $(P=0.001)$ and necrosis $(P=0.030)$, even if the intensity of these relationships resulted weaker ( $\mathrm{V}$ index was, respectively, equal to 0.398 and 0.289 ). No significant association was detected between remaining features and "pain" variable. 
Table 2 Histopathological features in tendon and subacromial bursa

\begin{tabular}{|c|c|c|c|c|c|c|}
\hline & \multicolumn{3}{|l|}{ Tendon } & \multicolumn{3}{|c|}{ Subacromial bursa } \\
\hline & Absent & Focal & Extended & Absent & Focal & Extended \\
\hline Disarray & $51(28.6 \%)$ & $117(64.4 \%)$ & $12(7 \%)$ & $123(68 \%)$ & $36(20 \%)$ & $21(12 \%)$ \\
\hline Oedema & $6(3.6 \%)$ & $156(88 \%)$ & $18(8.4 \%)$ & $9(4.6 \%)$ & $117(64.4 \%)$ & $54(31 \%)$ \\
\hline Neoangiogenesis & $84(46.4 \%)$ & $90(50 \%)$ & $6(3.6 \%)$ & $12(7 \%)$ & $108(61 \%)$ & $60(32 \%)$ \\
\hline Presence of inflammatory cells & $153(85.7 \%)$ & $21(10.7 \%)$ & $6(3.6 \%)$ & $81(44 \%)$ & $99(56 \%)$ & $0(0 \%)$ \\
\hline Fatty metaplasia & $138(76 \%)$ & $42(24 \%)$ & $0(0 \%)$ & $0(0 \%)$ & $0(0 \%)$ & $0(0 \%)$ \\
\hline Chondral metaplasia & $21(12 \%)$ & $99(56 \%)$ & $60(32 \%)$ & $150(84 \%)$ & $30(16 \%)$ & $0(0 \%)$ \\
\hline Fibrosis & $93(52.3 \%)$ & $84(46.5 \%)$ & $3(1.2 \%)$ & $72(39 \%)$ & $108(61 \%)$ & $0(0 \%)$ \\
\hline Necrosis & $123(67.8 \%)$ & $51(28.5 \%)$ & $6(3.6 \%)$ & $144(80 \%)$ & $36(20 \%)$ & $0(0 \%)$ \\
\hline Calcification & $168(93 \%)$ & $0(0 \%)$ & $12(7 \%)$ & $168(93 \%)$ & $12(7 \%)$ & $0(0 \%)$ \\
\hline Hypertrophy/hyperplasia & $141(78.6 \%)$ & $27(14.3 \%)$ & $12(7 \%)$ & $36(20 \%)$ & $63(36 \%)$ & $81(44 \%)$ \\
\hline
\end{tabular}
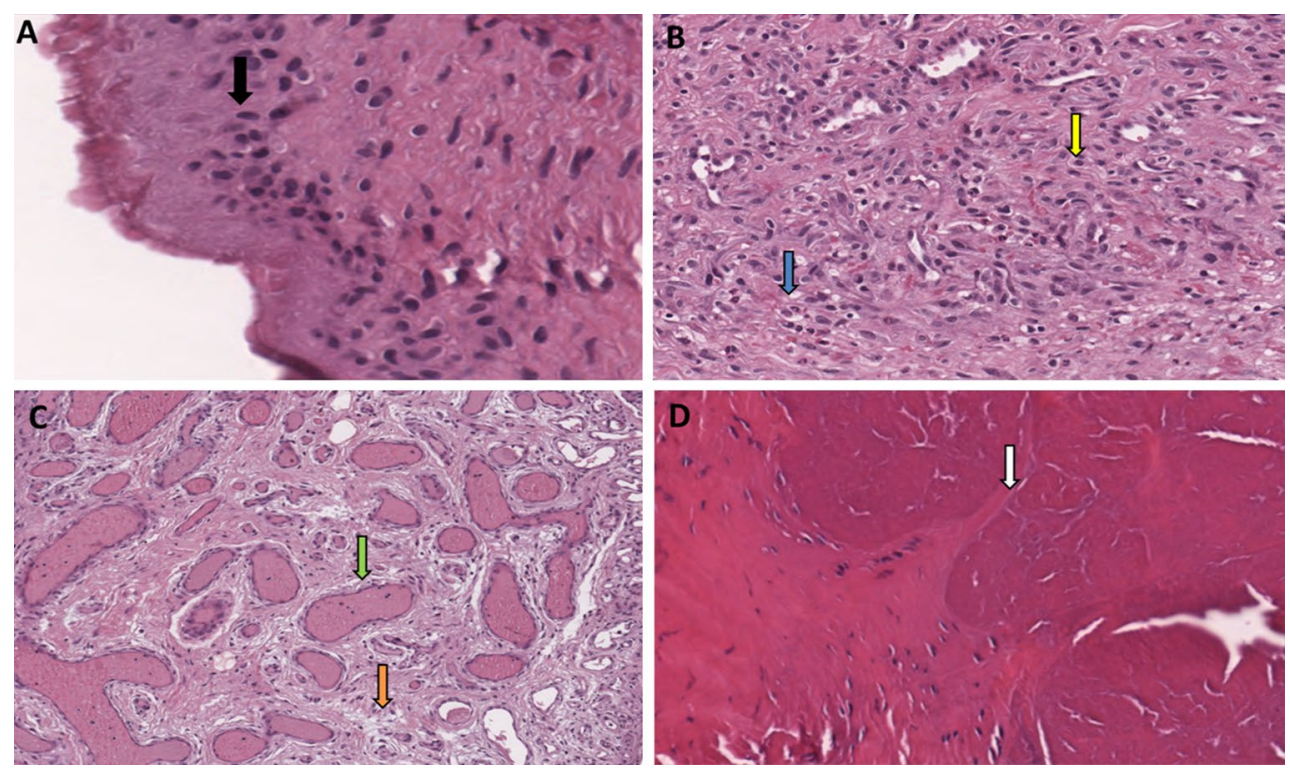

Fig. 1 a Tendon hypertrophy: connective tissue is covered by multilayered cubic synoviocytes (black arrow) (haematoxylin-eosin, magnification $\times 40$ ), b tendon inflammation: an intense inflammatory infiltrate of small lymphocytes (yellow arrow) and some neutrophils and eosinophils granulocytes (blue arrow) is clearly identified (haematoxylin-eosin, magnification $\times 20$ ), c bursal oedema: oedema

\section{Discussion}

The most important finding of the present study is the key role played by the subacromial bursa in the genesis of rotator cuff tear's pain. Pain is the most common symptom in patients with rotator cuff tears, and different studies have been conducted to explain its origin. Two main theories have been developed: the chemical theory and the mechanical one. Some studies support the chemical theory with the increased expression of cytokines, proinflammatory and growth factors (IL-18, IL-15, IL-6, MIF, TNF- $\alpha$, IL-1, (orange arrow) and numerous dilated and congested vessels covered with a monolayer of endothelial flat cells (green arrow) are present (haematoxylin-eosin, magnification $\times 20$ ), d bursal necrosis: connective tissue appears loose with multiple foci of necrosis (haematoxylin-eosin, magnification $\times 20$ )

COX-1, COX-2) that are found in both torn tendon and subacromial bursa $[10,18,22,27,33,34]$. The mechanical theory is focused in particular on the role of the LHB. In the presence of a full thickness rotator cuff tear, the exposed tendon of the LHB, due to its anterosuperior position, can become impinged against the acromial vault during forward flexion causing pain [21]. Moreover, also the simple degeneration of the LHB, often associated with rotator cuff tears, may be a source of persistent shoulder pain $[3,6,30,36]$.

However, to our knowledge, there is a lack of literature regarding factors which might influence the presence 


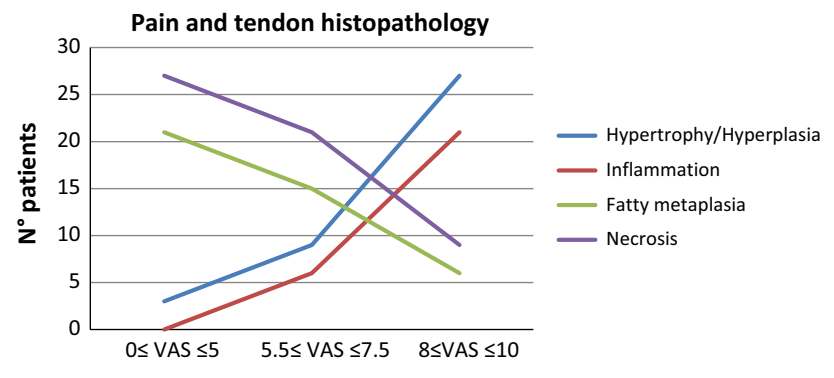

Fig. 2 Presence of inflammatory cells and hypertrophy/hyperplasia in tendon were significantly associated $(P<0.05)$ with pain. Instead pain decreased significantly $(P<0.05)$ with fatty metaplasia and necrosis

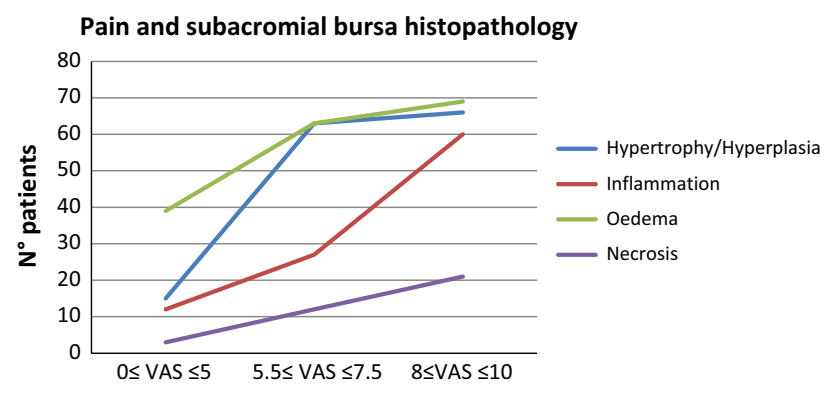

Fig. 3 Presence of hypertrophy/hyperplasia, inflammation, oedema and necrosis in the subacromial bursa were significantly associated $(P<0.05)$ with pain

of pain, and in particular, there are no studies up to date which could explain it with the histopathological findings observed in the torn tendon and subacromial bursa and with the clinical features of patients with rotator cuff tears.

The first aim of this study was to identify the main clinical features associated with pain, so to define an accurate profile of the painful rotator cuff tear. Female sex is significantly associated with higher pain levels: different studies have demonstrated that women have an increased sensitivity to pain $[7,20]$, and our paper supports this finding. Although the mechanisms underlying sex differences in pain have not been fully elucidated, both psychosocial and biological factors are involved [7, 20] with gonadal steroid hormones playing a major role [20]. In addition, from the results of our study, it seems that the Goutallier's grade is inversely associated with pain: the more is the level of muscle fatty infiltration, the less pain is experienced by the patient. The progressive loss of muscular tissue replaced by fat, and thus, the loss of $\mathrm{A} \delta$ and $\mathrm{C}$ fibres responsible for the conduction of pain signal from the periphery to the spinal cord could explain this relationship $[14,29]$. This finding is also in accordance with what we have found about the histopathological association between tendon fatty metaplasia and pain that is discussed below. Regarding the onset of symptoms, chronic rotator cuff tears are more painful compared with acute lesions. The fact that chronic tears actually are more painful seems to be in contradiction to the fact that fatty infiltration is inversely associated with pain, as it is well known that fatty infiltration represents an indicator of chronicity of the tear. The altered articular mechanics and the exposition of the LHB could explain this finding $[3,6,16,30,36]$. In fact, the presence of a chronic tear, although associated with an important fatty degeneration, typically results also in the exposition of the LHB. The consequence is that chronic tears compared with acute tears are more frequently associated with changes in the LHB that, as reported in the literature, are responsible for pain $[3,6,30,36]$. The importance of the LHB in chronic tears is also confirmed by the satisfactory results obtained with isolated biceps tenotomy performed in patients with irreparable rotator cuff tears [3]. In addition, advanced chronic tears, unlike acute tears, are often associated with a certain degree of proximal humeral migration and with a disruption of normal glenohumeral kinematics that may also represents a source of pain [16]. No statistically significant association was found between lesion size and pain and between age of patients and pain.

The second aim of this paper was to evaluate whether there was any relationship between specific histopathological features of the torn tendon and subacromial bursa and pain.

The presence of inflammation and hypertrophy/hyperplasia in both tendon and bursal tissue is directly associated with pain. This association can be explained by the increased production of cytokines, proinflammatory and growth factors. Surprisingly, it is in particular the subacromial bursa alterations, more than tendon, to be strongly associated with pain. As expected, also the presence of bursal oedema, a direct manifestation of bursal inflammation, was found to be associated with pain. Regarding the other tendon features, the presence of fatty metaplasia is inversely associated with pain. The same relationship was found for tendon necrosis although the strength of this association was not particularly prominent. These findings could be explained by the progressive loss of tendinous tissue, replaced by fat or destroyed by necrosis, and as a consequence by the loss of $\mathrm{A} \delta$ and $\mathrm{C}$ fibres responsible for the conduction of pain signal from the periphery to the spinal cord $[14,29]$. Contrary to what we found for tendon necrosis, we observed that pain increases in the presence of necrosis of the subacromial bursa. It is possible to hypothesize that the algogenic degradation products derived from the necrotic bursal tissue could be discharged into the bloodstream more easily compared with the necrotic tendon because of the better vascularization of the subacromial bursa [32]. 
Fig. 4 In order to reduce pain in rotator cuff tears, it is possible to use NSAIDs and surgically perform a wide bursectomy. However, if we look to the tendon healing process, we have to consider the detrimental effect of this class of drugs and the beneficial effect of bursal tissue on fibrillogenesis

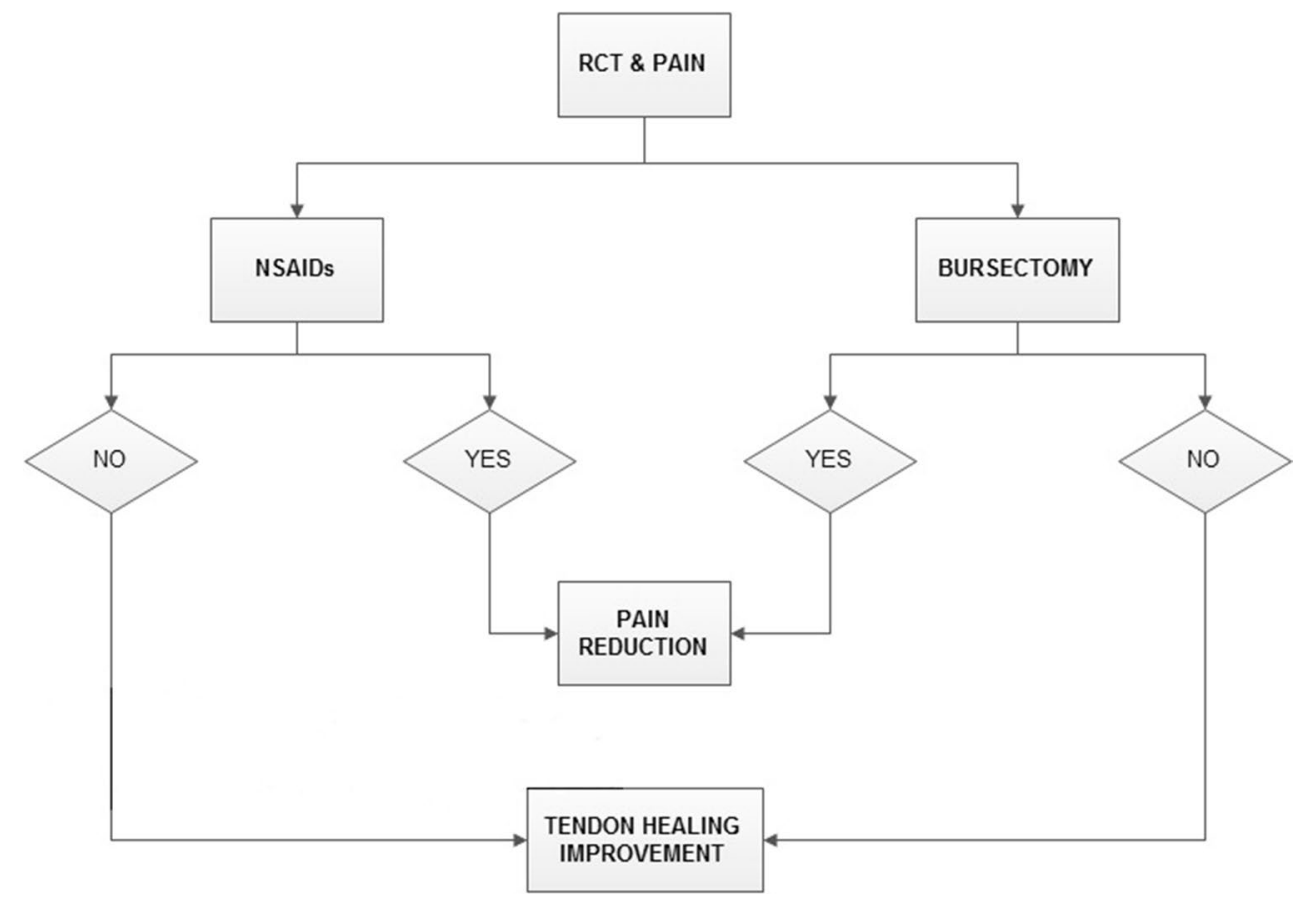

This study highlights the strong relationship existing between pain and inflammation confirming what had already been supposed from experimental laboratory studies $[18,22,27,33]$ and from the clinical practice $[4,17$, 23]. From the results of this study, it seems that the bursal tissue plays a key role in the genesis of pain. In particular, bursal tissue more than tendon could be considered as a "pain generator" and then as an enemy to fight.

This study has also several limitations. The biopsy specimens of tendon and subacromial bursa underwent histopathological analysis and were stained only with haematoxylin-eosin (H/E). No other specific stainings for nerves and neurological mediators of pain were performed. In addition, all the histopathological features were arbitrarily scored according to a semi-quantitative grading system. A more objective quantification of the histopathological features might be recommended.

The clinical relevance of this report for the management of rotator cuff tear is significant, concerning both surgical and pharmacological approaches to this pathology (Fig. 4). With the objective to reduce pain in patients surgically treated for repairing a rotator cuff tear, a wide bursectomy is commonly performed and our findings support this orientation. However, it is known that inflammation represents the first stage of the healing process [1, 11], and recent studies confirmed that the subacromial bursa plays a key role in the rotator cuff tear healing process in particular in elderly people and in large size lesions as it represents the major source of cells and blood vessels that are necessary for the repair [4, 5, 32]. Taking into account both the proreparative and algogenic roles of the subacromial bursa, we believe that bursectomy should be limited to the bare essentials for obtaining a good visualization of the tear pattern and a good mobility of the ruptured tendon and contemporarily preserve the bursal tissue as much as possible. The most common pharmacological treatment of shoulder pain in rotator cuff tear is the administration of non-steroidal anti-inflammatory drugs (NSAIDs) which are very effective in reducing pain $[2,17]$. However, several studies investigated the role of inflammation in promoting tendon fibrillogenesis and concluded that the administration of these drugs could have a detrimental effect on healing [11, $19,25,31]$. We believe that it is important to pharmacologically reduce pain but at the same time not to block the inflammation process. For this reason, it seems reasonable to use other classes of analgesic drugs rather than NSAIDs such as paracetamol and opioids.

\section{Conclusions}

This study defines the main clinical and histopathological features of painful rotator cuff tears. Clinically, rotator cuff tears are more painful in females, in chronic lesions and in the presence of a low muscle fatty degeneration. Histologically, rotator cuff tears are more painful in the presence of hypertrophy and inflammation of the tendon and in the presence of hypertrophy, inflammation, oedema and necrosis of the subacromial bursa.

In particular, a greater association of pain was observed with the histopathological changes in the bursa compared with those in the rotator cuff. Considering that the 
bursa plays also an essential role during the healing process (being a major source of cells and blood vessels), this "new" role of the subacromial bursa as pain generator has important repercussions in both pharmacological and surgical treatments of rotator cuff tears.

Acknowledgments The authors wish to thank Mrs. Tolani Lawson for reviewing the English.

Conflict of interest The authors declare that they have no conflict of interest.

\section{References}

1. Ahmad Z, Wardale J, Brooks R, Henson F, Noorani A, Rushton $N$ (2012) Exploring the application of stem cells in tendon repair and regeneration. Arthroscopy 28:1018-1029

2. Baydar M, Akalin E, El O, Gulbahar S, Bircan C, Akgul O, Manisali M, Torun Orhan B, Kizil R (2009) The efficacy of conservative treatment in patients with full-thickness rotator cuff tears. Rheumatol Int 29:623-628

3. Boileau P, Baqué F, Valerio L, Ahrens P, Chuinard C, Trojani C (2007) Isolated arthroscopic biceps tenotomy or tenodesis improves symptoms in patients with massive irreparable rotator cuff tears. J Bone Joint Surg Am 89:747-757

4. Chillemi C, Petrozza V, Garro L, Sardella B, Diotallevi R, Ferrara A, Gigante A, Di Cristofano C, Castagna A, Della Rocca C (2011) Rotator cuff re-tear or non-healing: histopathological aspects and predictive factors. Knee Surg Sports Traumatol Arthrosc 19:1588-1596

5. DeFranco MJ, Cole BJ (2009) Current perspectives on rotator cuff anatomy. Arthroscopy 25:305-320

6. Delle Rose G, Borroni M, Silvestro A, Garofalo R, Conti M, De Nittis P, Castagna A (2012) The long head of biceps as a source of pain in active population: tenotomy or tenodesis? A comparison of 2 case series with isolated lesions. Musculoskelet Surg 96:S47-S52

7. Fillingim RB, King CD, Ribeiro-Dasilva MC, Rahim-Williams B, Riley JL 3rd (2009) Sex, gender, and pain: a review of recent clinical and experimental findings. J Pain 10:447-485

8. Fish DE, Gerstman BA, Lin V (2011) Evaluation of the patient with neck versus shoulder pain. Phys Med Rehabil Clin N Am 22:395-410

9. Fuchs B, Weishaupt D, Zanetti M, Hodler J, Gerber C (1999) Fatty degeneration of the muscles of the rotator cuff: assessment by computed tomography versus magnetic resonance imaging. J Shoulder Elbow Surg 8:599-605

10. Garofalo R, Cesari E, Vinci E, Castagna A (2011) Role of metalloproteinases in rotator cuff tear. Sports Med Arthrosc 19:207-212

11. Gigante A, Specchia N, Rapali S, Ventura A, de Palma L (1996) Fibrillogenesis in tendon healing: an experimental study. Boll Soc Ital Biol Sper 72:203-210

12. Goutallier D, Postel JM, Bernageau J, Lavau L, Voisin MC (1994) Fatty muscle degeneration in cuff ruptures. Pre- and postoperative evaluation by CT scan. Clin Orthop Relat Res 304:78-83

13. Hyvönen P, Melkko J, Lehto VP, Jalovaara P (2003) Involvement of the subacromial bursa in impingement syndrome of the shoulder as judged by expression of tenascin- $\mathrm{C}$ and histopathology. $\mathrm{J}$ Bone Joint Surg Br 85:299-305
14. Ide K, Shirai Y, Ito H, Ito H (1996) Sensory nerve supply in the human subacromial bursa. J Shoulder Elbow Surg 5:371-382

15. Juel NG, Natvig B (2014) Shoulder diagnoses in secondary care, a one year cohort. BMC Musculoskelet Disord 15:89

16. Keener JD, Wei AS, Kim HM, Steger-May K, Yamaguchi K (2009) Proximal humeral migration in shoulders with symptomatic and asymptomatic rotator cuff tears. J Bone Joint Surg Am 91:1405-1413

17. Kijima H, Minagawa H, Nishi T, Kikuchi K, Shimada Y (2012) Long-term follow-up of cases of rotator cuff tear treated conservatively. J Shoulder Elbow Surg 21:491-494

18. Koshima H, Kondo S, Mishima S, Choi HR, Shimpo H, Sakai T, Ishiguro N (2007) Expression of interleukin-1beta, cyclooxygenase-2, and prostaglandin E2 in a rotator cuff tear in rabbits. $\mathbf{J}$ Orthop Res 25:92-97

19. Lee HJ, Kim YS, Ok JH, Lee YK, Ha MY (2015) Effect of a single subacromial prednisolone injection in acute rotator cuff tears in a rat model. Knee Surg Sports Traumatol Arthrosc 23:555-561

20. Manson JE (2010) Pain: sex differences and implications for treatment. Metabolism 59:S16-S20

21. Maynou C, Mehdi N, Cassagnaud X, Audebert S, Mestdagh $\mathrm{H}$ (2005) Clinical results of arthroscopic tenotomy of the long head of the biceps brachii in full thickness tears of the rotator cuff without repair: 40 cases. Rev Chir Orthop Reparatrice Appar Mot 91:300-306

22. Millar NL, Wei AQ, Molloy TJ, Bonar F, Murrell GA (2009) Cytokines and apoptosis in supraspinatus tendinopathy. J Bone Joint Surg Br 91:417-424

23. Neer CS 2nd (1983) Impingement lesions. Clin Orthop Relat Res 173:70-77

24. Perry SM, McIlhenny SE, Hoffman MC, Soslowsky LJ (2005) Inflammatory and angiogenic mRNA levels are altered in a supraspinatus tendon overuse animal model. J Shoulder Elbow Surg 14:79S-83S

25. Radi ZA, Khan NK (2005) Effects of cyclooxygenase inhibition on bone, tendon, and ligament healing. Inflamm Res 54:358-366

26. Rahme H, Nordgren H, Hamberg H, Westerberg CE (1993) The subacromial bursa and the impingement syndrome. A clinical and histological study of 30 cases. Acta Orthop Scand 64:485-488

27. Sakai H, Fujita K, Sakai Y, Mizuno K (2001) Immunolocalization of cytokines and growth factors in subacromial bursa of rotator cuff tear patients. Kobe J Med Sci 47:25-34

28. Snyder SJ (2003) Arthroscopic classification of rotator cuff lesions and surgical decision making. In: Snyder SJ (ed) Shoulder arthroscopy. Lippincott Williams \& Wilkins, Philadelphia, pp 201-207

29. Soifer TB, Levy HJ, Soifer FM, Kleinbart F, Vigorita V, Bryk E (1996) Neurohistology of the subacromial space. Arthroscopy 12:182-186

30. Szabó I, Boileau P, Walch G (2008) The proximal biceps as a pain generator and results of tenotomy. Sports Med Arthrosc 16:180-186

31. Tsai WC, Hsu CC, Chen CP, Chen MJ, Lin MS, Pang JH (2006) Ibuprofen inhibition of tendon cell migration and down-regulation of paxillin expression. J Orthop Res 24:551-558

32. Uhthoff HK, Sarkar K (1991) Surgical repair of rotator cuff ruptures. The importance of the subacromial bursa. J Bone Joint Surg Br 73:399-401

33. Voloshin I, Gelinas J, Maloney MD, O'Keefe RJ, Bigliani LU, Blaine TA (2005) Proinflammatory cytokines and metalloproteases are expressed in the subacromial bursa in patients with rotator cuff disease. Arthroscopy 21:1076.e1-1076.e9

34. Xu C, Yang X, Zhao J (2015) Arthroscopic treatment for synovial chondromatosis of the subacromial bursa associated with 
partial rotator cuff tear. Knee Surg Sports Traumatol Arthrosc 23:600-602

35. Yamamoto A, Takagishi K, Osawa T, Yanagawa T, Nakajima D, Shitara H, Kobayashi T (2010) Prevalence and risk factors of a rotator cuff tear in the general population. J Shoulder Elbow Surg 19:116-120
36. Zhang Q, Zhou J, Ge H, Cheng B (2015) Tenotomy or tenodesis for long head biceps lesions in shoulders with reparable rotator cuff tears: a prospective randomised trial. Knee Surg Sports Traumatol Arthrosc 23:464-469 